\title{
COMPARISON OF INTRADISCAL RESTORATIVE INJECTIONS AND INTRADISCAL ELECTROTHERMAL TREATMENT (IDET) in the Treatment of Low Back Pain
}

\author{
Richard Derby, MD, Björn Eek, MD, Sang-Heon Lee, MD, PhD, Kwan Sik Seo, MD, and Byung-Jo Kim, MD, PhD
}

This was a pilot study to test the potential effectiveness of intradiscal restorative injection therapy and compare with intradiscal electrothermal therapy (IDET). Thirty-five patients for intradiscal injection and seventy-four for IDET took part in the study. All patients had intractable chronic discogenic low back pain, confirmed by discogram study. Injection solution consisted of glucosamine and chondroitin sulfate combined with hypertonic dextrose and dimethylsulfoxide. Outcome was rated as $0-10$ on visual analog scale (VAS), satisfaction rate, and flare up before and after the

Discogenic low back pain (LBP) is due to various factors which alter the structure and integrity of the intervertebral disc. This pain is most persistent due to the low restorative capacity of disc tissue $(1,2)$. A variety of discogenic pain treatments have been introduced in last decade. Unfortunately, the most routinely used modalities for treatment of the intervertebral disc and disc-related problems do not address the underlying causes, but alleviate associated symptoms. Intradiscal electrothermal treatment (IDET) (3) is a recently developed approach which may induce thermal destruction of nociceptive fibers in the outer annulus fibrosus and is one of the treatment modalities providing symptomatic relief.

There is increasing interest in the development of more physiologic

From Spinal Diagnostics and Treatment Center, Daly City, CA, Division of Physical Medicine and Rehabilitation, School of Medicine, Stanford University, Stanford, CA, Orthopedic Medicine, Santa Barbara, CA, and Spinal Diagnostics and Treatment Center, Daly City, CA. Address Correspondence: Richard Derby, MD, 901 Campus Drive, Suite 310, Daly City, CA 94015. E-mail: rderby@spinaldiagnostics.com Support: There was no external funding in preparation of this manuscript.

Conflict of Interest: None procedures. Post-procedure, patients were followed from 6 months to 18 months. Pain relief was statistically significant for both procedures, but slightly better for injections (2.2 VAS) than for IDET (1.27 VAS). 47.8\% of IDET patients reported that they felt better, whereas $65.6 \%$ of injection patients reported this outcome. Among IDET patients, $35.8 \%$ reported they were worse, while no restorative injection patient reported worsening of pain. Post-procedure flare-up occurred more frequently after restorative injection (81\%) than after IDET (68.9\%) and was more severe (7.9 versus 6.1 VAS, respec-

treatment options for addressing the underlying causes of discogenic pain. Continued research has increased our histologic and physiologic knowledge of the intervertebral disc, affording several strategies such as artificial replacement and disc regeneration (4-8). Injections of restorative solution into the intervertebral disc can stimulate production of structural molecules and may possibly be used as a new treatment modality (911). Additionally, restorative injections in joints and tendons have been shown to be effective for treating connective tissue injury (12-14).

The goal of this study was to examine the effectiveness of restorative injections and compare these results with the effectiveness of IDET.

\section{Methods}

This study was retrospectively performed through the analysis of a prospectively collected database. Patients with putative chronic discogenic LBP, consecutively referred from primary care physicians and other clinicians during the period from January 2000 to October 2002, were eligible to participate. Intervertebral disc levels included in the study were determined in all patients preoperatively by tively). However, the duration of pain flareup was notably shorter for restorative injections (8.6 days) than for IDET (33.1 days). Biochemical intradiscal restorative injections may be useful to reduce pain and disability in patients with chronic discogenic low back pain, and have clinically similar efficacy to IDET, but with improved cost-benefit ratio. The results of this study indicate that controlled random prospective comparative studies need to be performed to establish the efficacy of this treatment.

Keywords: Intervertebral disc, injection, glucosamine, chondroitin sulfate, dextrose

independent correlative testing for back pain symptoms using provocative pressure-controlled manometric discography.

\section{Restorative Injection}

\section{Patient selection}

Thirty-five patients participated (17 men, 18 women, mean age: 42.0 years, range 25-60 years). All patients presented with chronic LBP and failed to respond or responded poorly to numerous treatment methods (e.g., physical therapy, multiple analgesics, ligament prolotherapy, laminectomy, fusion and IDET) and were being considered for additional surgical procedures. Twenty-four of the thirty-five patients had involvement of $>2$ discs. Seven of the 35 patients had previously undergone IDET at a single disc with varying, but generally poor, responses. Six of these seven patients received treatment to the disc previously treated by IDET. One patient received treatment to a different disc. Three patients had prior lumbar fusions at a single level and were symptomatic at additional levels. Three patients had laminectomies with persistent pain. Four patients, two of whom had a prior lumbar fusion, were disabled and had been incapacitated for $>1$ year. 


\section{Composition of injected solutions}

A compounding pharmacist using sterile technique and USP grade pharmaceuticals prepared the solutions which consisted of $0.5 \%$ chondroitin sulfate, $20 \%$ glucosamine hydrochloride, $12 \%$ DMSO and $2 \%$ bupivacaine. These concentrations were based upon the solubility and tolerance characteristics of the constituents. This solution was then mixed with equal parts non-ionic contrast and $50 \%$ dextrose at the time of injection.

\section{Injection protocol}

To avoid patient discomfort, the injection was performed during diagnostic discography. An intradiscal injection of 1-2 cc of solution was utilized at each involved disc level as determined by discography. Injections were performed using fluoroscopic guidance. If leakage of contrast into the epidural space was noted, the injection was terminated. Prophylactic antibiotics and standard discographic monitoring and sedation procedures were used.

\section{IDET}

\section{Patient selection}

Participants included 74 patients (32 men, 42 women, mean age: 41.57 years, age range: 17-62 years) who underwent IDET during the same period that restorative injections were performed. All patients presented with LBP of discogenic origin established via discography of the lumbar spine within the past 6 months. All patients failed to respond to previous conservative treatment including nerve blocks, with non-focal neurologic examination, disc protrusion $\leq 2 \mathrm{~mm}$, single level pathology, and positive discogram with annular tear. Subjects with allergy to any contrast media, iodine, or cephalosporin antibiotics were excluded.

\section{Procedure}

Prior to injection a fluoroscopic examination of the spine was performed to confirm segmentation and determine the appropriate level for needle placement. Using standard discographic practices, a 17-gauge introducer was placed into the center of the disc. Position was confirmed by fluoroscopy in oblique, antero-posterior (AP), and lateral views. A navigable intradiscal catheter with a 6 -cm active electrothermal tip (SpineCATH ${ }^{\circledR}$, Oratec Interventions, Menlo Park, CA) was then advanced and passed diametrically across the nucleus pulposus until it contacted the inner antero-lateral annulus. With continued insertion the electrode deflected circumferentially back towards the insertion side, with its circuitous route encompassing the inner perimeter of the annulus. After satisfactory catheter placement, an ORA-50 ${ }^{\mathrm{TM}} \mathrm{S}$ ElectroThermal ${ }^{\mathrm{TM}}$ Spine Generator was attached and gradually heated to $90{ }^{\circ} \mathrm{C}$ over 16.5 minutes. Once coagulation was complete, cefazolin antibiotic and $0.5 \%$ bupivacaine were administered intradiscally for antimicrobial prophylaxis and post-procedure analgesia, respectively.

\section{Exclusion Criteria}

We excluded patients with unstable medical conditions, instability and spondylolisthesis, severe spinal stenosis, and reduced disc height $>50 \%$. Patients who could not speak English were also excluded for accuracy of outcome.

\section{Other Treatments}

Following the procedure, patients were given a lumbar support brace to deter movements that might elevate intradiscal pressure (e.g., forward bending) and were instructed to forego intense physical training for a period of 6 months. In the first month, permitted activities included walking and gentle leg stretches. Over the next 5 months, the intensity of exercise was gradually increased until patients engaged in normal activities by 6 months.

\section{Assessment of Outcome}

Subjects were instructed on the scoring of the visual analogue scale (VAS, 0-10) used pre- and post-procedure. Patients were also examined using our evaluation sheet, which included patient's subjective impressions of outcome such as satisfaction and improvement, pain distribution and regional improvement, activity level, etc. Post-procedure, patients were followed from 6-18 months. Follow-up was done face-to-face during clinic visits or by telephone. Evaluation sheets were recorded at each follow-up.

\section{Data Analysis}

Data entry was performed by a person independent from the study. Statistical analyses were executed using SPSS/PC+ software ${ }^{\circledR}$ (SPSS, Inc., Chicago, Illinois).

\section{RESULTS}

The IDET procedure was performed on 74 patients and 35 patients received intradiscal injections. There were no significant differences in the essential demographic characteristics of the groups. Average ages in IDET and injection groups were 41.6 and 42.0 years, respectively. Types of insurance and gender ratios did not differ significantly. Follow-up duration in IDET patients was longer (15.5 months) than in the injection group (7.7 months).

Among patients treated by restorative injection, five required epidural corticosteroid injections 1-3 weeks after receiving intradiscal injections due to a significant flare-up of pain. There were significant differences in the occurrence, severity, and duration of flare-up. Post-procedure flare-up occurred more frequently after restorative injection $(81 \%)$ than after IDET (68.9\%) and was more severe (7.9 versus 6.1 VAS, respectively). However, the duration of pain flare-up was notably shorter for restorative injections (8.6 days) than for IDET (33.1 days).

Pain relief was statistically significant for both procedures, but slightly better for injections (2.2 VAS) than for IDET (1.27 VAS), although the difference was only marginally significant $(\mathrm{p}=0.01)$. Patients receiving injections were significantly more satisfied with the results of treatment. Only $47.8 \%$ of IDET patients reported that they felt better, whereas $65.6 \%$ of injection patients reported this outcome. Among IDET patients, $35.8 \%$ reported they were worse, while no restorative injection patient reported worsening of pain.

\section{DisCUSSION}

Oral glucosamine and chondroitin sulfate enhance proteoglycan synthesis. These agents have been used in multiple clinical trials and have generally been found effective and safe in the treatment of peripheral joint osteoarthritis (15-17). There is evidence that glucosamine and chondroitin sulfate synergistically enhance the natural hypermetabolic repair response of chondrocytes and retard the enzymatic degradation of cartilage (18). These properties encouraged us to explore their potential use in degenerative disc disease. Because the blood supply to the intervertebral disc is poor and oral glucosamine and chondroitin sulfate do not clearly benefit patients with LBP 
(19), we elected to perform a pilot study using intra-discal injectable glucosamine and chondroitin sulfate combined empirically with other agents (DMSO and hypertonic dextrose) in an attempt to promote a reparative response in the intervertebral disc.

Our data show statistically significant improvements in pain and disability in a group of patients with multi-level involvement and refractory pain, suggesting that this approach is worthy of further pursuit and refinement. All 35 patients who received restorative injection experienced varying degrees of post-injection pain. In most cases this could be controlled with oral analgesics and was limited to 72 hours of moderate to severe pain. One patient required a tapering dose of oral corticosteroids for 1 week post-treatment. Five patients required epidural corticosteroids due to temporary exacerbations of pain. All patients were treated prophylactically with antibiotics at the time of each intradiscal injection, and there were no instances of disc space infection or other serious complications. There were no instances of skin rashes, systemic reactions, hypotension or allergic reactions noted with any of the injections.

We hypothesize that the reduction in pain and disability seen in patients treated with restorative injection results from favorable alterations in the biochemical milieu of the intervertebral disc. However, we have no direct evidence that this is the case. The present study was neither blinded nor randomized, and we cannot rule out a placebo effect as a major contributor to improvement. Further studies, including serial magnetic resonance imaging scans, are clearly needed to address this important question.

Biochemical restorative injection for the treatment of degenerative disc disease is controversial. However, more specific use of proliferative agents, combined with the technical advantages of fluoroscopically guided anatomic site verification, is a reasonable prospect (20). Mooney recently suggested that restorative treatment is no longer at the periphery, and seems to be at the frontier of a justifiable, rational treatment with significant potential to avoid destructive procedures (20). While the clinical advantages of restorative injections are not pronounced, these data suggest that such injections may offer a plausible early option when the relatively high cost of IDET is considered.

\section{Conclusion}

Intradiscal injections of a glucosamine and chondroitin sulfate solution combined with dextrose and DMSO may provoke indirect stimulation of connective tissue healing. This approach may significantly decrease pain and disability in patients with refractory chronic LBP. Clinical efficacy is similar to that of IDET, but with an improved cost-benefit ratio. The results of this study indicate that controlled randomized, prospective comparative studies need to be performed to establish the efficacy of intradiscal restorative injection.

\section{ACKNOWLEDGMENTS}

The authors wish to thank Todd M. Billeci at the University of California, San Francisco for editing the manuscript.

\section{Author Affiliation: \\ Richard Derby, MD \\ Medical Director \\ Spinal Diagnostics and Treatment Center \\ 901 Campus Drive, Suite 312 \\ Daly City, CA 94015 \\ E-mail: rderby@spinaldiagnostics.com}

Björn Eek, MD

Orthopedic Medicine

2927 De La Vina

Santa Barbara, CA 93105

E-mail: eek@spinaldiagnostics.com

Sang-Heon Lee, MD, PhD

Physiatrist \& Research Physician

Spinal Diagnostics and

Treatment Center

901 Campus Drive, Suite 312

Daly City, CA 94015

E-mail: lee@spinaldiagnostics.com

\section{Kwan Sik Seo, MD}

Physiatrist \& Research Physician

Spinal Diagnostics and

Treatment Center

901 Campus Drive, Suite 312

Daly City, CA 94015

E-mail: seo@spinaldiagnostics.com

Byung-Jo Kim, MD, PhD

Neurologist \& Research Physician

Spinal Diagnostics and

Treatment Center

901 Campus Drive, Suite 312

Daly City, CA 94015

E-mail:kim@spinaldiagnostics.com

\section{References}

1. Diwan AD, Parvataneni HK, Khan SN et al. Current concepts in intervertebral disc restoration. Orthop Clin North Am 2000; 31:453-464.

2. Ochi M, Uchio Y, Tobita $M$ et al. Current concepts in tissue engineering technique for repair of cartilage defect. Artif Organs 2001; 25:172-179.

3. Saal JA, Saal JS. Intradiscal electrothermal treatment for chronic discogenic low back pain: prospective outcome study with a minimum 2-year follow-up. Spine 2002; 27:966-973.

4. Nishimura K, Mochida J. Percutaneous reinsertion of the nucleus pulposus. An experimental study. Spine 1998; 23:1531-1538.

5. Lee CK, Langrana NA, Parsons JR et al. Development of a prosthetic intervertebral disc. Spine 1991; 16:S253-255.

6. Ray CD. The PDN prosthetic disc-nucleus device. Eur Spine J 2002; 11 2:S137-142.

7. Kotani Y, Abumi K, Shikinami Y et al. Artificial intervertebral disc replacement using bioactive three-dimensional fabric: Design, development, and preliminary animal study. Spine 2002; 27:929-935.

8. Sato M, Asazuma T, Ishihara M et al. An experimental study of the regeneration of the intervertebral disc with an allograft of cultured annulus fibrosus cells using a tissue-engineering method. Spine 2003; 28:548-553.

9. Hacket G. Ligament and Tendon Relaxation Treated by Prolotherapy. 3rd ed. Charles C. Thomas, Springfield, 1958.

10. Mathews R, Miller M, Bree S. Treatment of mechanical and chemical lumbar discopathy by dextrose $25 \%$. J Minim Invasive Spinal Tech 2001; 1:58-61.

11. Klein RG, Eek BC, O'Neill CW et al. Biochemical injection treatment for discogenic low back pain: a pilot study. Spine J 2003; 3:220-226.

12. Reeves KD, Hassanein KM. Long-term effects of dextrose prolotherapy for anterior cruciate ligament laxity. Altern Ther Health Med 2003; 9:58-62.

13. Reeves KD, Hassanein K. Randomized, prospective, placebo-controlled doubleblind study of dextrose prolotherapy for osteoarthritic thumb and finger (DIP, PIP, and trapeziometacarpal) joints: Evidence of clinical efficacy. I Altern Complement Med 2000; 6:311-320.

14. Tsatsos G, Mandal R. Prolotherapy in the treatment of foot problems. I Am Podiatr Med Assoc 2002; 92:366-368.

15. Deal CL, Moskowitz RW. Nutraceuticals as therapeutic agents in osteoarthritis. The role of glucosamine, chondroitin sulfate, and collagen hydrolysate. Rheum Dis Clin North Am 1999; 25:379-395.

16. McAlindon TE, LaValley MP, Gulin JP et al. Glucosamine and chondroitin for treatment of osteoarthritis: a systematic quality assessment and meta-analysis. JAMA 2000; 283:1469-1475. 
17. Hungerford DS, Jones LC. Glucosamine and chondroitin sulfate are effective in the management of osteoarthritis. I Arthroplasty 2003; 18:5-9.

18. Lippiello L, Woodward J, Karpman R et al. In vivo chondroprotection and metabolic synergy of glucosamine and chondroitin sulfate. Clin Orthop 2000; 381:229-240.

19. Leffler CT, Philippi AF, Leffler SG et al. Glucosamine, chondroitin, and manganese ascorbate for degenerative joint disease of the knee or low back: a randomized, double-blind, placebo-controlled pilot study. Mil Med 1999; 164:85-91.

20. Mooney V. Prolotherapy at the fringe of medical care, or is it the frontier? Spine J 2003; 3:253-254. 